\title{
Development of a small-diameter and high-resolution industrial endoscope with CMOS image sensor
}

\author{
Lei Yang ${ }^{\mathrm{a}}$, Meng Chen ${ }^{\mathrm{a}}$, Qian Zhu ${ }^{\mathrm{a}}$, Tong Yang ${ }^{\mathrm{a}}$, Chao Wang ${ }^{\mathrm{b}}$, Hongbo \\ $\mathrm{Xie}^{\mathrm{a}, *}$ \\ ${ }^{a}$ School of Precision Instrument and Opto-electronics Engineering, Tianjin University \\ Key Laboratory of Optoelectronics Information Technology, Ministry of Education, \\ Tianjin, 300072, China; \\ ${ }^{b}$ School of Engineering and Digital Arts, University of Kent, Canterbury CT2 7NT, \\ United Kingdom \\ *Email: hbxie@tju.edu.cn
}

\begin{abstract}
In the industrial field, an endoscope is typically employed for the observation and inspection of internal defect and corrosion of an engine and chemical plant. Current industrial optical endoscopes normally use CMOS sensor which offers best performance as an image pickup device. Here we report experimental demonstration of an industrial endoscope modality consisting of a $5 \mathrm{~mm}$ diameter rigid tube and $1280 \times 960$ pixels CMOS camera, which enables accurate diagnosis of small-scale industrial components with hollow shape. As a proof of concept, we successfully perform arc-surface imaging at a resolution of $57 \mathrm{lp} / \mathrm{mm}$ and $\pm 30^{\circ}$ field of view. Finally, potentially applying the wave-front coding method to extend the depth of field of endoscopic system is comprehensively discussed.
\end{abstract}

Keywords: Miniature optical endoscope, CMOS sensor, High resolution PACS codes: 07.07.Hj, 42.15Eq., 42.30.Va

MSC codes: 78A05

\section{Introduction}

In recent years, there has widely been employed an optical endoscope capable of non-destructively inspecting a component or an interior of a turbine in various modern industrial regions [1]. In general, an industrial endoscope has an unbending insertion section, which is inserted into a to-be-inspected 
space, such as the inside of a pipe [2]. Small-diameter endoscopes are highly needed for inspection in the narrow space of the diverse industrial components, however, an essential challenge still exists in conventional apparatus to create high-resolution vision $[3,4,5]$. The present article demonstrates an optical endoscope consisting of one $5 \mathrm{~mm}$-diameter rigid tube and CMOS (Complementary metal oxide semiconductor) sensor unit, which has the capability of providing imaging resolution better than $57 \mathrm{lp} / \mathrm{mm}$.

In pursuit of affording an endoscope apparatus which is made small in diameter, small-scale optical elements with different shapes and sizes are introduced that try to collect and distribute light in a specifically desired way. A set of variables that can change to improve performance, such as curvature, thickness as well as index of refraction, are properly optimized for satisfying the requirement of system. We also find that a CMOS device compared favorably with the CCD (Charge-coupled device) currently used to register visible light as an image sensor. In addition, CMOS sensors offer numerous other advantages including higher speed, low energy consumption and user-friendly interface $[6,7]$.

Apart from traditional optical imaging, there are several proposals having been directed to reduce the size of endoscopic device while ensuring excellent insertion and operation properties. An alternative for performing an optical image of internal surfaces is utilizing single-mode fiber to deliver, focus, scan and collect light beam, which has inner diameter of approximately $1 \mathrm{~mm}$ [8]. However, fiber probe endoscopes commonly suffer from some inherent drawbacks that involves fragility and difficult to install. Other miniature endoscopes only $2-5 \mathrm{~mm}$ in diameter will integrate photoacoustic imaging $[9,10]$, ultrasonic $[9,11]$ or spectrally coding [12] approaches, which fall short in complex configuration and high cost. Therefore, a traditional optical industrial endoscope with its unique merits of stability, simple structure, easy assemble, cost-effective and less maintenance is also extremely attractive in inspection procedures to allow internal visualization.

This article discusses fundamental and practical considerations of an optical industrial endoscope with commercial CMOS sensor. Sec. 2 explains the theory for determining the spatial resolution and depth of field (DOF) in optical systems. Sec. 3 shows an optical design model and simulation results that reveal the performance of the proposed system in detail. In Sec. 4, we describe the experimental configuration and procedure that has been developed and implemented to demonstrate the potential of this modality. In Sec. 5, practical limitation of this industrial endoscope is discussed, and it 
is shown that DOF can be significantly extended using newly-designed phase mask. A conclusion is drawn in Sec. 6.

\section{Principle and theory}

The resolution of an industrial endoscope is defined as the smallest resolvable distance between two objects, which can still be distinguished by the CMOS camera as separate entities. In our modality, spatial resolution of optical system is given by

$$
\sigma_{s}=\frac{1.22 \lambda}{D / f^{\prime}}
$$

where $\lambda$ is the wavelength of incident light, $\mathrm{D}$ is the diameter of aperture, and $f^{\prime}$ is the focal length. Matching optical resolution of endoscope $\sigma_{c}$ to a specific CMOS camera is also crucial, which ensures minimum pixel density necessary to adequately capture all of the optical data form the endoscope. Therefore, the optical resolution of endoscopic system is found to be

$$
\frac{1}{\sigma_{t}}=\frac{1}{\sigma_{s}}+\frac{1}{\sigma_{c}}
$$

where $\sigma_{c}$ represent the resolution of CMOS sensor.

In optical system, DOF refers to the distance between objects that appear acceptably sharp in an image, which can be approximated to be [13]

$$
\delta l=\frac{2 D f^{\prime} l^{2} Z^{\prime}}{D^{2} f^{\prime 2}-l^{2} Z^{\prime 2}}
$$

where $l$ denotes the object distance, and $Z^{\prime}$ denotes maximum diameter of spot on the detector.

\section{Design and simulation}

The modality of industrial endoscope under consideration is schematically shown in Fig. 1. This system mainly consists of an objective lens, rod lenses, magnifying lenses and a protective glass, and its secondary image structure is beneficial to achieve required working length. When a bunch of beam ray enters the objective lens, it will be reflected by the optical prism to the tilting lense with $90^{\circ}$ turning. Two rod lenses with cylindrical shape are rotated and located in the correct orientation to conduct beam ray and efficiently extend 


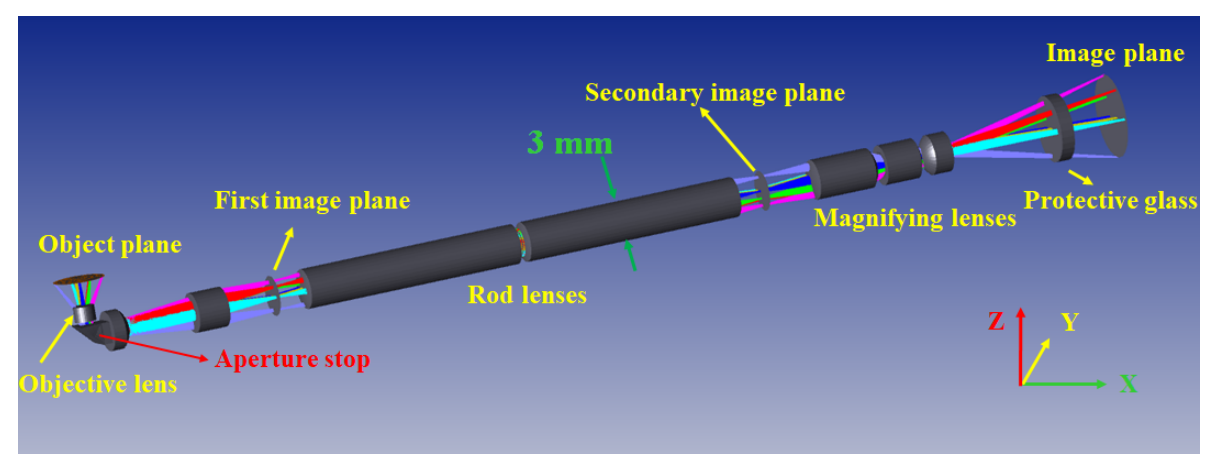

Figure 1: Model of industrial endoscope designed in Zemax. The effective focal length $f^{\prime}=3.21 \mathrm{~mm}$, and aperture diameter $\mathrm{D}=0.35 \mathrm{~mm}$.

the optical path, which provides longer working distance for endoscope. The magnifying lenses with convex shape are used to produce a magnified image that is matched with the dimension of CMOS camera. An extra glass is attached to the screen of the CMOS camera to protect against damage. The purposive field of view (FOV) of $60^{\circ}$ in X-direction and $48.6^{\circ}$ in Y-direction are defined to satisfy the 4:3 image aspect ratio. Moreover, owing to large FOV always accompanying with severe optical distortion, we have to make a trade-off between FOV and optical distortion, and therefore the optimized FOV is limited at $60^{\circ}$.

The system can be quantitatively evaluated by the simulation results that are depicted in Fig. 2. More concretely, Fig. 2(a) shows the calculated tangential and sagittal modulation transfer function (MTF) curves with various FOVs, which truly indicates the image performance of resolution achieves to $60 \mathrm{lp} / \mathrm{mm}$. The graphs of spot diagram show the ray aberrations of optical design are comparable to the size of diffraction limited spots (see Fig. $2(\mathrm{~b}))$. We also use the distortion grid to find the system distortion that is less than $4 \%$ in the border, as can been see in Fig. 2(c). In addition, the lateral chromatic aberration leaded by obliquely incident beam is minimized to $2 \mu \mathrm{m}$ in the FOV of $60^{\circ}$. From what has been discussed above, we may reasonably draw at the conclusion that the optical system holds the potential to be operated with high performance. 


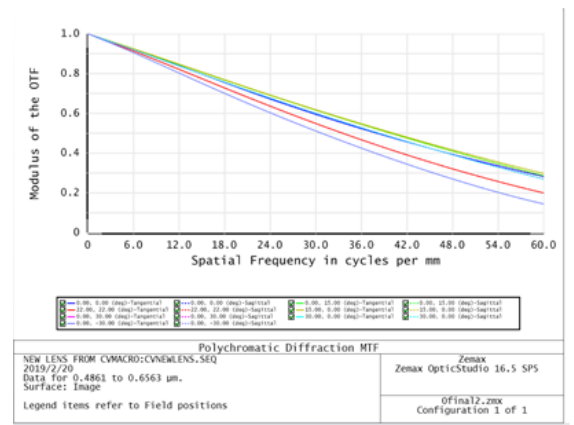

(a)

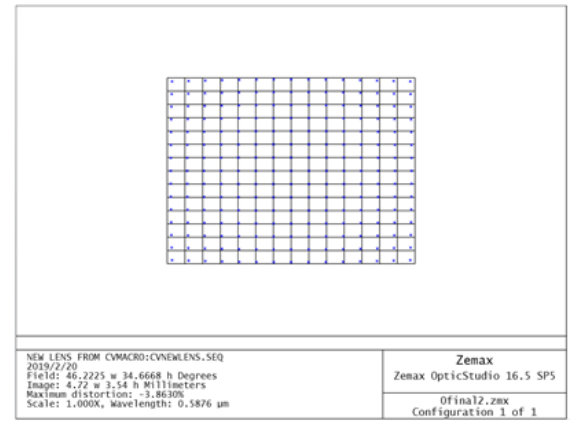

(c)

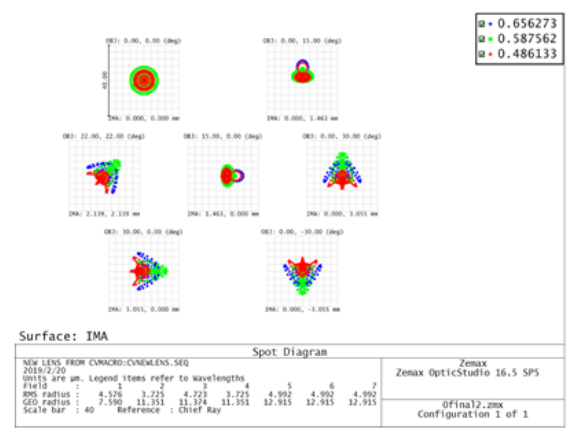

(b)

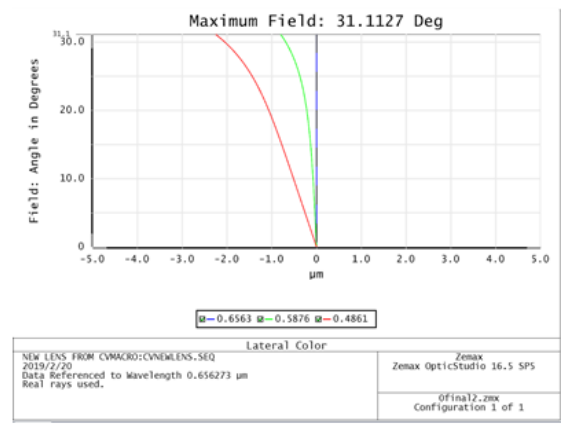

(d)

Figure 2: Evaluation results of the proposed system. (a) MTF. (b) Spot diagram. (c) Distortion grid. (d) Lateral chromatic aberration. 


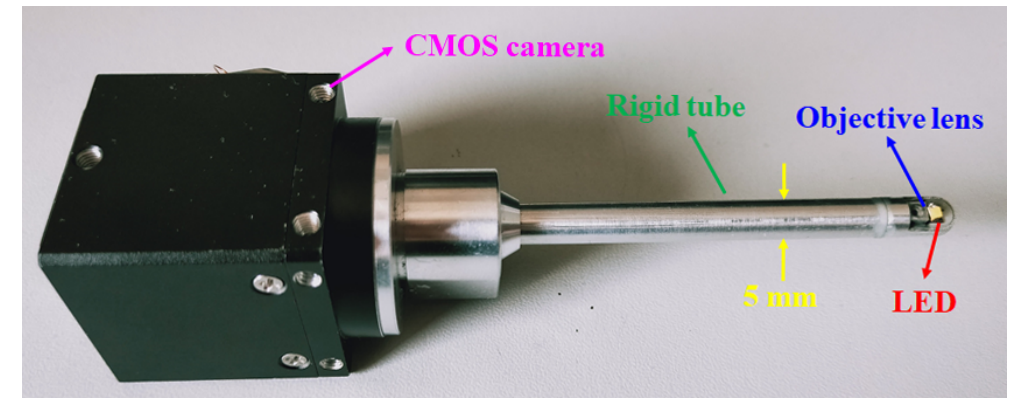

Figure 3: Assembled industrial endoscope.

\section{Experiment and results}

This endoscopic structure demands components with high precision and reliability, which were manufactured by the specialized Duralumin. Correct alignment of the optical system is also paramount important to ensure maximum brightness and resolution of endoscopic device. As shown in Fig. 3, the endoscope has a camera portion and an elongate scope portion extending from the camera portion to a tip. This small diameter rigid tube has 5 basic sections that make up the outer shell and structure of the scope. These sections are constructed differently but all provide a protective outer layer and a hollow inside. The hollow areas inside contain the delicate internal systems.

We employ a monochromatic $1 / 3^{\prime \prime}$ CMOS camera to register visible light as an electronic signal, which consists of $1280 \times 960$ pinned photodiode pixels with $3.75 \mu \mathrm{m}$ pitch and programmable conversion gain. This image sensor expertly handles applications that demand detection, quantification and speed. The industrial endoscope also has an illuminating section, in which a chip LED is used to emit 500 - $700 \mathrm{~nm}$ light with $150^{\circ}$ viewing angle. A piece of homogenization film is placed on the top surface of LED for the sake of generating uniform illumination light, which is operated at voltage of $3.3 \mathrm{~V}$. According to the provided parameters including $l=2 \mathrm{~mm}$ and $Z^{\prime}$ $=7.5 \mu \mathrm{m}$, the theoretical endoscopic resolution and DOF are $71 \mathrm{lp} / \mathrm{mm}$ and $50 \mu \mathrm{m}$, respectively.

Resolution chart of 1951 USAF is inserted into the endoscopic imaging system to certify its accuracy and performance, whose result is shown in Fig. 4. The result provides the image of red circle marked in the chart that exhibits the tested endoscope is capable of resolving details to a greater degree 


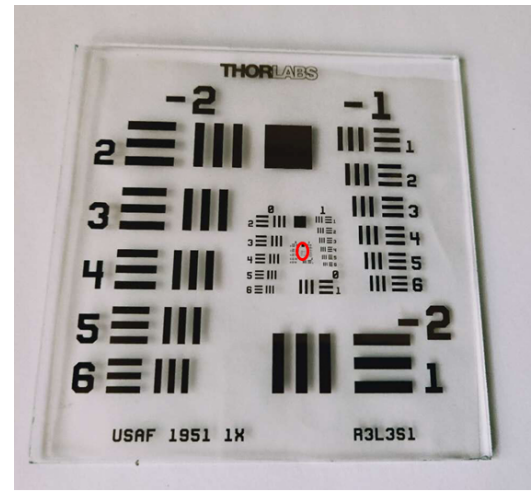

(a)

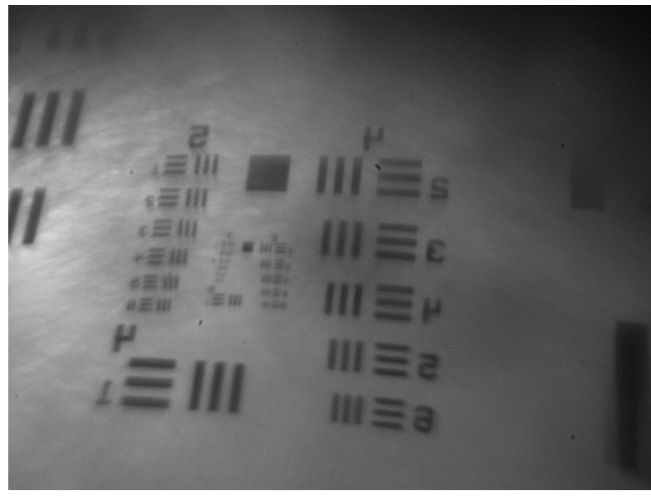

(b)

Figure 4: Testing result of endoscope. (a) 1951 USAF resolution test chart. (b) Image of aiming part that is marked by red circle in this chart. The best captured resolution is 57 $\mathrm{lp} / \mathrm{mm}$, which is close to the theoretical value of $71 \mathrm{lp} / \mathrm{mm}$.

than $57 \mathrm{lp} / \mathrm{mm}$. A bit fuzzy for the captured image is because the endoscopic device is designed for arc-surface imaging instead of flat-surface imaging. Additionally, we also used a rolling paper with ink printed characters as the tested target, and the clear images of character with $2 \times 1 \mathrm{~mm}^{2}$ verify the ability of the modality to recognize tiny unit with FOV of $60^{\circ}$ (see Fig. 5).

Testing for non-destructive visual inspection of industrial component with 40-mm-long and hollow cavity is shown in Fig. 6. We stuck an enameled wire with 0.02-mm-diameter to imitate the defects existed on the inner surface of the component. Apparently, the enameled wire were clearly observed using the assembled endoscope, especially for the burrs on the wire. In the practical inspection procedure, it will demand clamping this device and rotating it with a given speed to cover the whole area of component in $360^{\circ}$. Consequently, this approach is expected to be valuable for industrial applications in which high resolution is required.

\section{Discussion}

In this endoscopic modality, the DOF, defined as the range of distance over which a subject appears in-focus, is limited to a small range due to the fixed properties of the employed lens modules. More particularly, our modality should be appropriate for the components with small diameter, and therefore the attempt to increase the object distance for improving the 


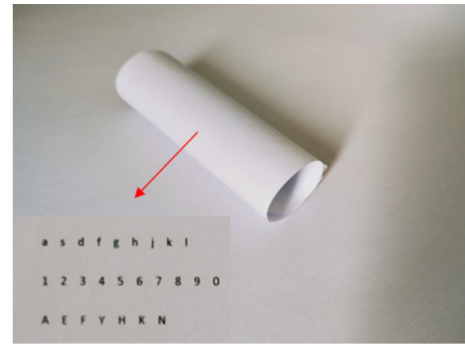

(a)

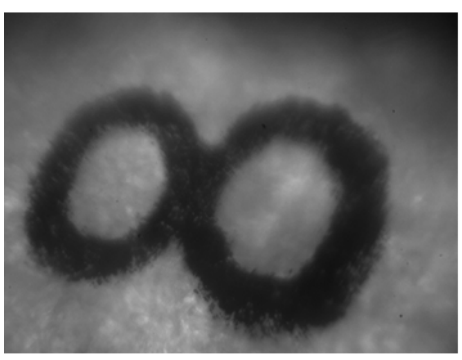

(c)

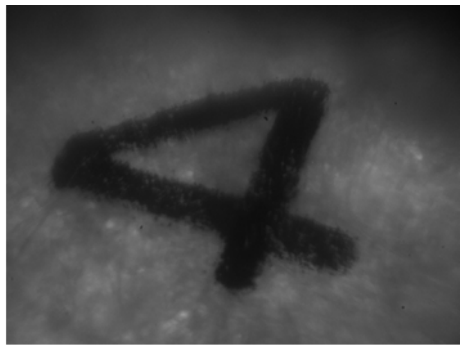

(b)

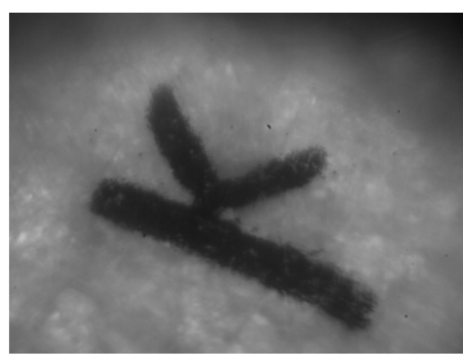

(d)

Figure 5: Images of the paper pipe with printed characters inside. (a) Paper pipe. The inset map shows the inner wall of the pipe. (b) Image of figure "4". (c) Image of figure "8". (d) Image of character of "k".

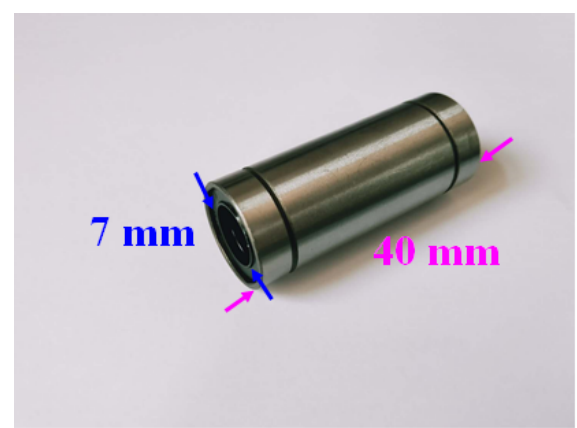

(a)

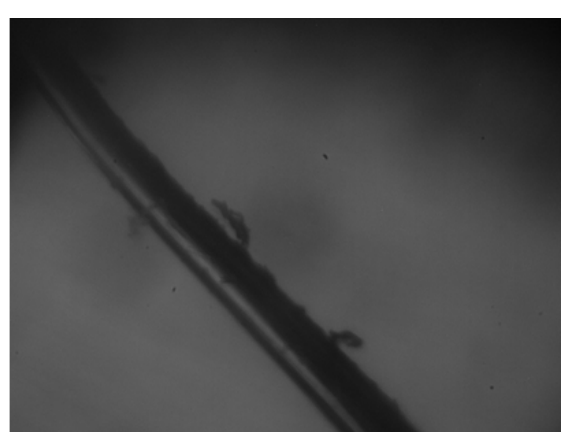

(b)

Figure 6: Imaging interior of one representive industrial component. (a) Industrial component. (b) A piece of enameled wire stuck on the inner surface of component. 

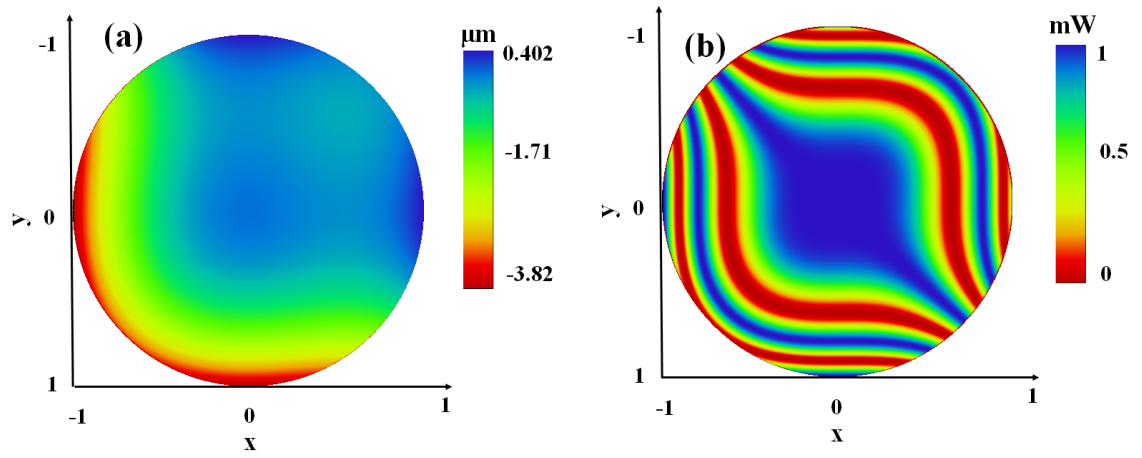

Figure 7: Phase mask with normalized spatial coordinates. (a) Structure of the phase mask. The scale bar represents the thickness variation. (b) Interferogram of the phase mask with incident parallel rays. The scale bar represents the power of incident light with unit of $\mathrm{mW}$.

DOF is not available. Wave-front coding is a prevalent strategy used to appreciably improve the DOF without sacrificing the resolution $[14,15,16]$, which combines a fabricated phase mask and digital signal processing in a fundamental manner $[17,18,19]$. Owing to the phase mask occupying the whole pupil aperture, and therefore power of propagating light and spatial resolution is completely preserved [20].

One of our goals here is to design a high-order phase mask that makes the MTF and point spread function (PSF) of initial system having low sensitivity to focus errors. The altered MTF and PSF will cause the medium images with specialized and well-defined blurs, which could be eliminated with digital signal processing. The phase mask is correctly placed at the position of aperture stop to accurately manipulate the incident beam ray (see Fig. 1), which is equal to introduce extra parameter in the pupil function to eliminate the defocus value. The detailed information of the optimized phase mask is illustrated in Fig. 7, including the surface structure and interferogram. The function of phase mask is given by

$$
f(x, y)=A\left(x^{2}+y^{2}\right)+B\left(x^{3}+y^{3}\right)
$$

where $A=-0.01221$ and $B=0.04037$ represent the surface coefficients.

In Fig. 8, a series of MTF curves are carried out to estimates the features of the system under the behaviour of optimized phase mask, which are almost consistent with object distance varying. In addition, the PSFs are 

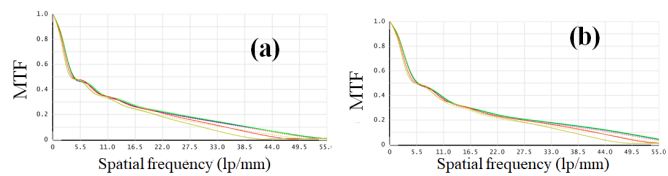

(d)
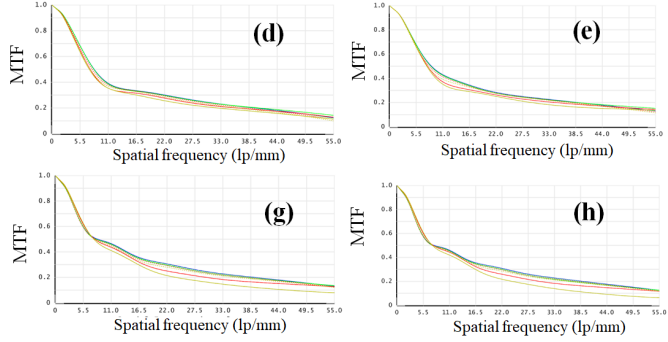

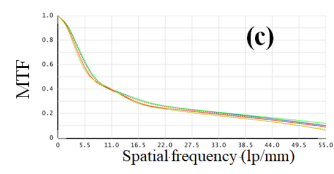

(f)
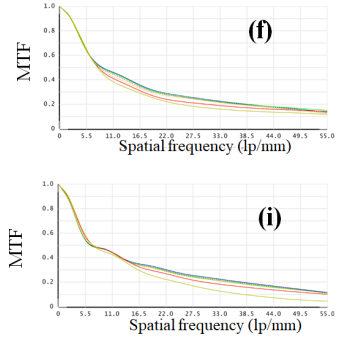

$-\quad$ Tangential MTF in $0^{\circ} \mathrm{FoV}$
$-\quad$ Sagittal MTF in $0^{\circ} \mathrm{FoV}$

- Tangential MTF in $12^{\circ} \mathrm{FoV}$

Sagittal $\mathrm{MTF}$ in $12 \mathrm{~F}^{\circ} \mathrm{F}$

- Tangential MTF in $24^{\circ}$ FoV

Sagittal MTF in $24^{\circ} \mathrm{FoV}$

Tangential MTF in $30^{\circ} \mathrm{Fol}$

Sagittal MTF in $30^{\circ} \mathrm{FoV}$

Figure 8: MTF curves in various object distances. (a) $1.8 \mathrm{~mm}$. (b) $2 \mathrm{~mm}$. (c) $2.4 \mathrm{~mm}$. (d) $2.8 \mathrm{~mm}$. (e) $3.2 \mathrm{~mm}$. (f) $3.6 \mathrm{~mm}$. (g) $4 \mathrm{~mm}$. (h) $4.2 \mathrm{~mm}$. (i) $4.5 \mathrm{~mm}$. The blue solid line, green solid line, red solid line and yellow solid line represent the tangential MTFs in $0^{\circ}$, $12^{\circ}, 24^{\circ}, 30^{\circ} \mathrm{FOV}$, respectively. The blue dotted line, green dotted line, red dotted line and yellow dotted line represent the sagittal MTFs in $0^{\circ}, 12^{\circ}, 24^{\circ}, 30^{\circ} \mathrm{FOV}$, respectively.

simulated and depicted in Fig. 9, and the compared results reveal the wavefront coded PSFs keep invariant in different object distances. Therefore, it is not difficult to draw the conclusion that an extension in the DOF from 1.8 to $4.5 \mathrm{~mm}$ could be implemented in the future work [14, 17]. On the other hand, perfect matching between PSF and recovery digital algorithm is highly needed, otherwise, the ultimate image probably losses initial resolution.

\section{Summary}

Traditional optical endoscope is an indispensable tool for inspecting the inner wall of diverse apparatuses, which is comprised of an insertion rigid tube with optical lens, CMOS camera sensor and illumination device. With its attribute to perform high resolution measurements, the proposed miniature endoscope device is also expected to be useful for a wide range of applications such as fingerprint detection, shape reconstruction and many more. A commercial CMOS camera was recommended to this inspection application, as it guarantees higher availability and a better price-performance ratio. Nevertheless, as a result of rigid tube being unable to bend, this rigid endoscope is lack of adaptability compared to the flexible scope.

Nowadays, industrial endoscope is a rapidly growing market and the demand for small-sized device to access narrow spaces is really possible to 

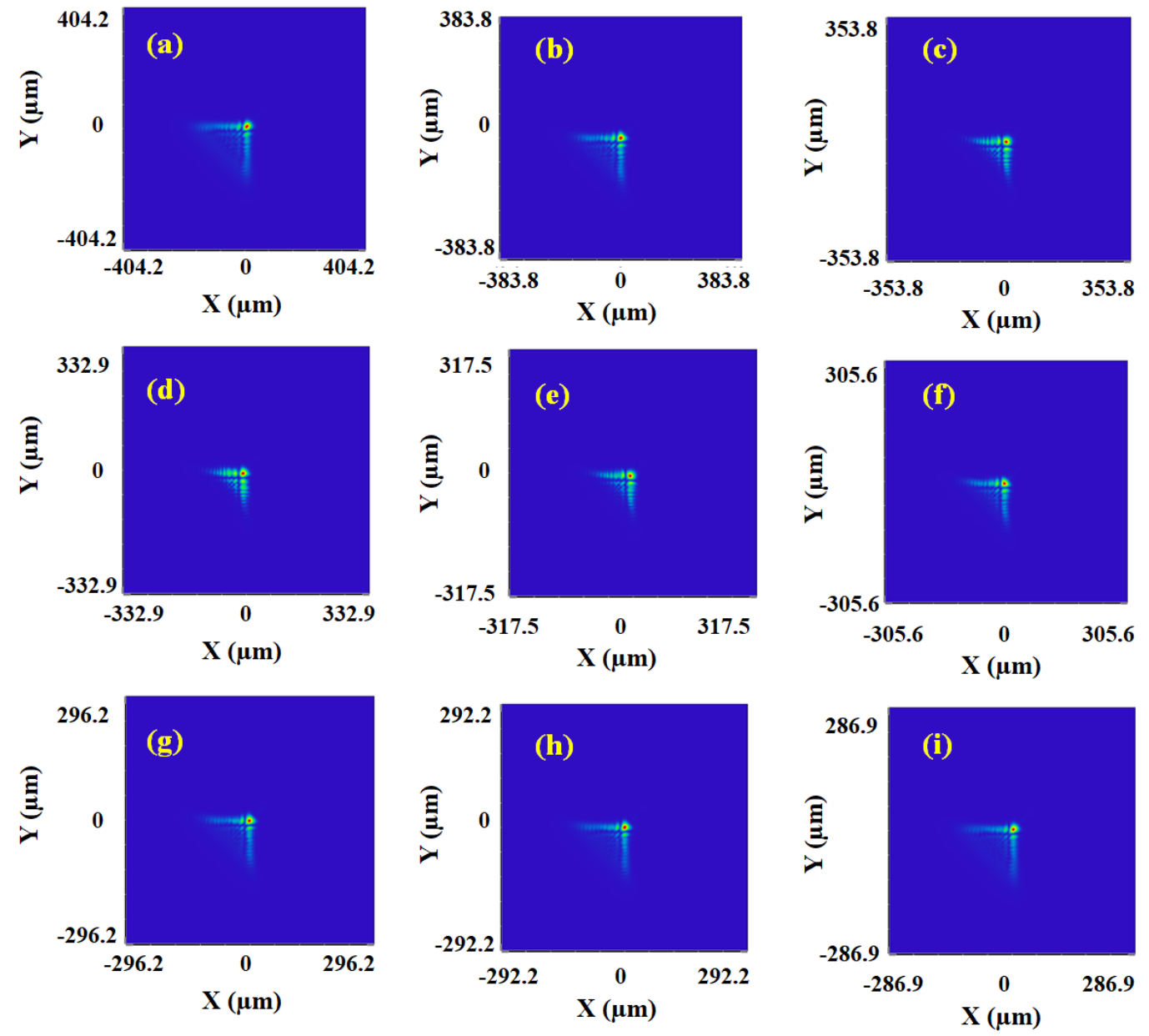

Figure 9: PSFs in various object distances. (a) $1.8 \mathrm{~mm}$. (b) $2 \mathrm{~mm}$. (c) $2.4 \mathrm{~mm}$. (d) 2.8 mm. (e) $3.2 \mathrm{~mm}$. (f) $3.6 \mathrm{~mm}$. (g) $4 \mathrm{~mm}$. (h) $4.2 \mathrm{~mm}$. (i) $4.5 \mathrm{~mm}$. The relative irradiances in each pictures are roughly same. 
increase. The proposed cost-effective visualization solution with high level of performance and image quality offers valuable strategy into the operation of endoscopy technology. Furthermore, conventional optical endoscopic modules have their intrinsic limitation because of small DOF, and it would lead to low tolerance of misalignments and bring inaccuracy to the comprehensive vision [21]. Inserting a phase mask in the aperture stop of endoscopic optical system has the capability of apparently enlarging the DOF, which allows inspection procedures more effective and stable. Future works lie in the interests of developing an ultra-thin optical endoscope with high-resolution and large DOF which is applicable for vast industrial and scientific areas.

[1] M. Stellingwerff and J. Breen. Applications of optical and digital endoscopy. Proceedings 2nd EAEA-Conference (Vienna), pages 55-68, 1995.

[2] M. Garcia and V. Gruev. Optical characterization of rigid endoscopes and polarization calibration methods. Optics Express, 25:15713-15728, 2017.

[3] M. Tadao, H. Ryunosuke, M. Wataru, E. Masayoshi, and H. Yoichi. A high-resolution endoscope of small diameter using electromagnetically vibration of single fiber. IEEJ Transactions on Sensors and Micromachines, 129:399-404, 2009.

[4] D. H. Cho, B. Lee, S. C. Chung, G.-R. Tack, and J. H. Yi. Development of a high-resolution flexible ultrathin endoscope using a new optical design and a contact method. IFMBE Proceedings, 14:1338-1340, 2007.

[5] G. S. Neel, R. L. Kau, S. F. Bansberg, and D. Lal. Comparison of 3 $\mathrm{mm}$ versus $4 \mathrm{~mm}$ rigid endoscope in diagnostic nasal endoscopy. World Journal of Otorhinolaryngology - Head and Neck Surgery, 3:32-36, 2017.

[6] R. B. Erarslana, U. Adiyana, S. Z. Lulec, S. Olcer, Y. Temiz, Y. Leblebici, H. Torunc, and H. Urey. Design and characterization of micromachined sensor array integrated with cmos based optical readout. Sensor and Actuators A: Physical, 215:44-50, 2014.

[7] M. Vatteronif, D. Covi, C. Cavallotti, L. Clementel, P. Valdastri, A. Menciassi, P. Darioa, and A. Sartori. Smart optical cmos sensor for endoluminal applications. Sensor and Actuators A: Physical, 162:297303, 2010. 
[8] G. J. Tearney, S. A. Boppart, B. Bouma, M. E. Brezinski, N. J. Weissman, J. F. Southern, and J. G. Fujimoto. Single mode fiber-optic catheter/endoscope for optical coherence tomography. Optics Letters, 21:543-545, 1996.

[9] J. M. Yang, R. Chen, C. Favazza, J. J. Yao, C. Y. Li, Z. L. Hu, Q. F. Zhou, K. K. Shung, and L. V. Wang. A 2.5-mm diameter probe for photoacoustic and ultrasonic endoscopy. Optics Express, 20:23944-23935, 2012.

[10] R. Ansari, E. Z. Zhang, A. E. Desjardins, and P. C. Beard. All-optical forward-viewing photoacoustic probe for high-resolution 3d endoscopy. Light: science applications, 7:75, 2018.

[11] S. R. Friedberg and J. Lachter. Endoscopic ultrasound: Current roles and future directions. World Journal of Gastrointestinal Endoscopy, 9:499-505, 2017.

[12] G. J. Tearney, M. Shishkov, and B. E. Bouma. Spectrally encoded miniature endoscopy. Optics Letters, 27:412-414, 2002.

[13] A. Lipson, S. G Lipson, and H. Lipson. Optical Physics. Cambridge University Press, 2010.

[14] Jr. E. R. Dowski and W. T. Cathey. Extended depth of field through wave-front coding. Applied Optics, 34:1859-1866, 1995.

[15] J. Yang, L. Gong, Y. Shen, and L. V. Wang. Synthetic bessel light needle for extended depth-of-field microscopy. Applied Physics Letters, 113:181104, 2018.

[16] P.Zammit, A. R. Harvey, and G. Carles. Extended depth-of-field imaging and ranging in a snapshot. Optica, 1:209-216, 2014.

[17] H. B. Xie, L. R. He, L. Yang, C. S. Mao, M. Zhu, M. Zhao, J. L. Li, and T. Yang. An extended depth-of-field imaging system with a nonrotationally symmetric phase mask. Review of Scientific Instruments, 89:103101, 2018.

[18] D. Calvetti, S. Morigi, L. Reichel, and F. Sgallari. Tikhonov regularization and the l-curve for large discrete ill-posed problems. Journal of Computational and Applied Mathematics, 123:423-446, 2000. 
[19] H. B. Xie, Y. P. Su, M. Zhu, L. Yang, S. S. Wang, X. B. Wang, and T. Yang. Athermalization of infrared optical system through wavefront coding. Optics Communications, 441:106-112, 2019.

[20] A. Castro and J. Ojeda-Castaneda. Asymmetric phase masks for extended depth of field. Applied Optics, 43:3474-3479, 2004.

[21] H. S. Chen and Y. H. Lin. An endoscopic system adopting a liquid crystal lens with an electrically tunable depth-of-field. Optics Express, 21:18079-18088, 2013. 